\title{
Disability, anxiety and depression associated with medication-overuse headache can be considerably reduced by detoxification and prophylactic treatment. Results from a multicentre, multinational study (COMOESTAS project)
}

Cephalalgia

2014, Vol. 34(6) 426-433

(C) International Headache Society 2013

Reprints and permissions:

sagepub.co.uk/journalsPermissions.nav DOI: $10.1177 / 0333102413515338$ cep.sagepub.com

@SAGE

\section{Bendtsen', SB Munksgaard', C Tassorelli', G Nappi ${ }^{2}$, Z Katsarava ${ }^{3}$, M Lainez ${ }^{4}$, JA Leston ${ }^{5}$, R Fadic ${ }^{6}$, S Spadafora ${ }^{7}$, A Stoppini ${ }^{8}$, R Jensen' and the COMOESTAS Consortium}

\begin{abstract}
Objective: The objective of this article is to investigate whether headache-related disability, depression and anxiety can be reduced by detoxification and prophylactic treatment in patients with medication-overuse headache $(\mathrm{MOH})$.

Methods: Patients with $\mathrm{MOH}$ were included from six centres in Europe and Latin America in a seven-month cohort study. Before and six months after treatment, the degree of disability was measured by the Migraine Disability Assessment (MIDAS) questionnaire, while anxiety and depression were measured by the Hospital Anxiety and Depression Scale (HADS).

Results: A total of 694 patients with $\mathrm{MOH}$ were included, of whom 492 completed the study. Headache days were reduced by $58.4 \%$ from 23.6 to 9.8 days per month at six months $(p<0.001)$. The MIDAS score was reduced by $57.1 \%$ from baseline 59.9 to $25.7(p<0.001)$. Number of patients with depression was reduced by $50.7 \%$ from 195 to 96 and number of those with anxiety was reduced by $27.1 \%$ from 284 to 207 (both $p<0.00$ I).

Conclusions: Disability, depression and anxiety were considerably reduced in patients with $\mathrm{MOH}$ by detoxification and prophylactic treatment. This emphasises the urgent need for increased awareness about avoiding overuse of headache medications and demonstrates that not only headache frequency but also disability are remarkably improved by adequate intervention.
\end{abstract}

\section{Keywords}

Medication-overuse headache, detoxification, disability, depression, anxiety, management

Date received: 23 April 20I3; revised: 28 May 20I3; II August 20I3; 3 September 20I3; 20 September 20I3; accepted: 27 September 2013

\footnotetext{
'Danish Headache Centre, Department of Neurology, Glostrup Hospital, Faculty of Health Sciences, University of Copenhagen, Denmark

${ }^{2}$ Headache Science Center, IRCCS Neurological Institute C. Mondino

Foundation, University of Pavia, Italy

${ }^{3}$ Department of Neurology, University Hospital of Essen, Germany

${ }^{4}$ Foundation of the Valencian Community, University Clinical Hospital, Spain

${ }^{5}$ Foundation for Combating Neurological Diseases of Childhood,

Argentina

${ }^{6}$ Pontificia Universidad Católica de Chile, Chile
}

\footnotetext{
${ }^{7}$ Universidad Isalud, Argentina

${ }^{8}$ Bioengineering and Medical Informatics Consortium, Italy

COMOESTAS Consortium: M Allena ${ }^{2}, \mathrm{G}$ Sances $^{2}, \mathrm{G}$ Sandrini ${ }^{2}, \mathrm{~F}$ Blandini ${ }^{2}$, M Rapsch ${ }^{3}$, B Lopez ${ }^{4}, M T$ Goicoichea ${ }^{5}$, D Cerquetti ${ }^{5}$, B Shand $^{6}$, M Osa ${ }^{7}$, M Pagani $^{8}$

Corresponding author:

Lars Bendtsen, Danish Headache Centre, Department of Neurology, Glostrup Hospital, Ndr. Ringvej, Faculty of Health Sciences, University of Copenhagen, Glostrup 2600, Denmark.

Email: lars.bendtsen@regionh.dk
} 


\section{Introduction}

Medication-overuse headache $(\mathrm{MOH})$ is a common, disabling and costly disease (1). It is very important to recognise medication overuse because it is potentially treatable and in principle also preventable (2). Several studies have demonstrated a high success rate just by providing information or other simple actions (3-5). Even patients previously regarded as treatment resistant by specialists can be effectively treated by a structured, multidisciplinary treatment programme $(2,6)$.

Most of the mentioned studies have focused on headache-specific measures such as headache days and medication overuse as primary endpoints. From the patient's perspective, the degree of disability that she or he suffers is often equally or even more important. This has been less often examined in $\mathrm{MOH}$ and only in single-centre studies (2,7-11). The Migraine Disability Assessment (MIDAS) questionnaire has been demonstrated to be a reliable measure of migraine disability and has been proposed as a useful indicator of the burden of migraine and, additionally, as an endpoint in assessing treatment efficacy (12-14).

Frequent headache and medication overuse are associated with anxiety and depression (8) but the causeeffect relationship is still unclear. The Hospital Anxiety and Depression Scale (HADS) is a well-validated tool for screening depression and anxiety not only in psychiatric patients but also in somatic patients (15-17). There have been mixed results as to whether baseline MIDAS and HADS scores can predict the success of detoxification, i.e. reduction of intake of analgesics to an amount no longer causing $\mathrm{MOH}$, of patients with MOH $(3,10,18)$.

The aim of the present study was to investigate whether headache-related disability, anxiety and depression can be reduced by an intervention approach consisting of mandatory detoxification plus optional prophylactic treatment in patients with $\mathrm{MOH}$ in a large multicentre, multinational treatment study.

\section{Subjects and methods}

\section{Subjects}

The present study was part of a larger study with the main aim to compare relapse rates six months after detoxification between $\mathrm{MOH}$ patients using an electronic or a paper headache diary. Patients referred to six headache centres in Europe (Italy, Denmark, Germany and Spain) and Latin America (Argentina and Chile) were recruited consecutively from August 2008 to February 2009. At a baseline visit before entering the detoxification programme, patients were interviewed and generally and neurologically examined by a physician experienced in headache. Patients who could meet inclusion criteria were identified. The primary headache type was assessed based on a detailed history.

Inclusion criteria were a diagnosis of $\mathrm{MOH}$, age between 18 and 65 years, and capability to fill in paper and/or electronic diaries (19). Presence of anxiety and depression was allowed unless considered so severe that there was a need for pharmacological treatment and that the including physician recommended that the patient should undergo psychiatric evaluation before detoxification. Exclusion criteria were a current diagnosis of co-existent, significant and complicating medical or psychiatric illnesses, significant overuse of 'pure' opioids (patients overusing combination drugs containing opioids are allowed), benzodiazepines, and barbiturates, overuse of alcohol and other drugs of addiction, current treatment with migraine prophylactic drugs, inefficacy of previous adequate detoxification programmes, inability to provide reliable information about medical history and pregnancy or breast feeding.

\section{Methods}

At visit 0 (see Figure 1), i.e. one month before detoxification, patients were informed individually about $\mathrm{MOH}$ and the treatment programme by a specialised headache nurse or physician and began keeping a headache diary.

At visit 1 (Day 0), the headache diary was checked and patients who still fulfilled inclusion criteria were included in the study. Based on the national health care service regulations, detoxification was performed either as inpatient detoxification (daily hospitalisation or regular hospitalisation) or as outpatient detoxification. Patients were provided with prescriptions for prophylactic medication, rescue medication and symptomatic medications. On Day 1, the overused drugs were abruptly discontinued and prophylactic medication was initiated if deemed appropriate by the physician. From Days 1 to 7 , patients were allowed three days with either naproxen $500 \mathrm{mg}$ up to two times a day or acetaminophen $1 \mathrm{~g}$ up to three times a day. Additionally, the patients were allowed to take rescue medication: promethazine $25 \mathrm{mg}$ or levomepromazine $25 \mathrm{mg}$ up to three times a day, and metoclopramide $20 \mathrm{mg}$ up to three times a day. From Day 8, the patients were allowed two days/week with symptomatic medications prescribed by the physician at the baseline visit. Symptomatic medications included simple analgesics, nonsteroidal anti-inflammatory drugs (NSAIDs), triptans, and combination analgesics, all except the previously overused drugs. All medications were 


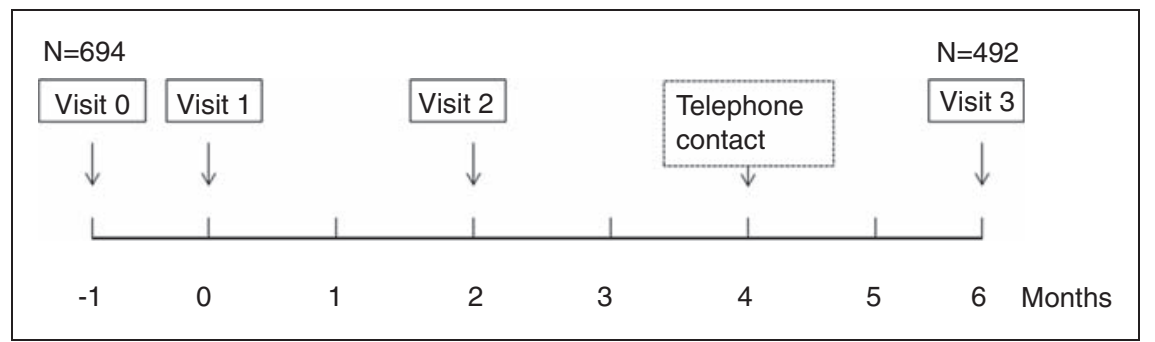

Figure I. Study plan. Following baseline visit 0 , patients with a retrospective history of medication-overuse headache ( $\mathrm{MOH})$ were asked to fill in a headache diary for one month and return to the centre for visit I. At visit I, if $\mathrm{MOH}$ was confirmed by the diary data, patients were enrolled in the study, detoxified and scheduled for a subsequent visit 2 after two months. During visit 2 clinical conditions and diary data were evaluated and the patients were scheduled for the final visit 3 after another four months. A telephone contact was performed halfway between visits 2 and 3 .

administered as tablets except for metoclopramide, which were suppositories. Prophylactic treatment was chosen based on the patients' primary headache, efficacy and side effects of previous treatments, co-morbid disorders, and preferences and was started from Day 1.

At visit 2 (two months after start of detoxification), the patients saw a physician or headache nurse, who collected the diary and checked the medication use. The physician evaluated and adjusted, if necessary, the prophylactic and symptomatic medications. Four months after starting detoxification the patients were interviewed by telephone by a physician or headache nurse checking that they had not relapsed to medication overuse. Patients were seen at the headache centres six months after detoxification (visit 3). Patients continued keeping the headache diary until six months after starting detoxification.

Disability was graded according to MIDAS score as minimal (0-5), mild (6-10), moderate (11-20) or severe $(21+)$ as described by Stewart et al. (13).

Presence of depression and anxiety at baseline was defined as having a HADS depression score or anxiety score of 8 or more (15). No specific interventions were made for anxiety or depression during the study.

The study was approved by the local ethics committees from all centres except from Denmark, where the local ethics committee stated that approval was not necessary because the study did not foresee any new pharmacological treatment. Participants gave informed consent before taking part.

\section{Statistics}

The primary outcome in the present study was the change in MIDAS score from baseline to six months after withdrawal. The secondary outcomes were the changes in HADS depression and anxiety scores from baseline to six months after withdrawal. Finally, we compared headache outcomes between the patients with high and those with low MIDAS and HADS scores at baseline. Data are presented as means with standard deviations in brackets. We used the Student's paired and independent $t$-tests for data following a normal distribution. For data with skewed distribution, we used the Mann-Whitney $U$-test or Wilcoxons signed ranks test. For dichotomous data, we used Fisher's exact test for samples less than five and the $\chi^{2}$-test for larger samples.

Power calculations were performed on the basis of variables of interest for the main study. The primary outcome variable was relapse rate six months after withdrawal. According to the literature the average relapse rate six months after withdrawal is $30 \%$. It was assumed that relapse rate could be reduced to $10 \%$ by using an electronic headache diary. It was calculated that 300 patients per group (standard treatment vs treatment using electronic diary) were needed to detect the assumed difference of $10 \%$ with the power of $80 \%$ and alpha error of $5 \%$. It was furthermore assumed that approximately $15 \%$ of patients would be lost to follow-up. Therefore a total number of 690 patients should be enrolled.

\section{Results}

A total of 694 patients with $\mathrm{MOH}$ were evaluated and $492(70.9 \%)$ completed this part of the study, i.e. patients for whom there were complete data on headache days, drug days for all seven months, and MIDAS and HADS both from baseline and at six months' follow-up. Baseline characteristics for the 492 completers and the 202 non-completers are presented in Table 1. A total of $94.1 \%$ of the patients were started on preventive therapy with $32.7 \%$ being treated with anti-epileptics, $28.7 \%$ with tricyclic antidepressants, $18.5 \%$ with beta-blockers and $14.2 \%$ with calcium channel blockers. 
Table I. Baseline characteristics of all included patients, $n=694$. The primary headache type was assessed based on history.

\begin{tabular}{|c|c|c|c|}
\hline & $\begin{array}{l}\text { Completers, } \\
n=492\end{array}$ & $\begin{array}{l}\text { Non-completers, } \\
n=202\end{array}$ & $p$ \\
\hline Age & $40.7(12.1)$ & $38.5(12.5)$ & 0.03 \\
\hline Gender, female & 390 (79\%) & 159 (79\%) & 0.92 \\
\hline \multicolumn{4}{|l|}{ Primary headache type } \\
\hline Migraine without aura & 391 (79\%) & 149 (75\%) & 0.15 \\
\hline Migraine with aura & $87(18 \%)$ & $28(14 \%)$ & 0.26 \\
\hline Chronic migraine & $42(9 \%)$ & $17(9 \%)$ & 1.00 \\
\hline Episodic tension-type headache & $70(14 \%)$ & $32(16 \%)$ & 0.56 \\
\hline Chronic tension-type headache & $92(19 \%)$ & $52(26 \%)$ & 0.04 \\
\hline Other headache & $10(3 \%)$ & $2(1 \%)$ & 0.74 \\
\hline Headache frequency (days/month) & $23.6(6.1)$ & $23.8(5.9)$ & 0.60 \\
\hline Duration of chronic headache (years) & $5.1(6.8)$ & $5.8(7.1)$ & 0.24 \\
\hline Duration of $\mathrm{MOH}$ (years) & $4.3(5.8)$ & $4.5(5.6)$ & 0.65 \\
\hline \multicolumn{4}{|l|}{ Type of overuse } \\
\hline Simple analgesics & $224(46 \%)$ & $99(50 \%)$ & 0.35 \\
\hline Combination-analgesics & $119(24 \%)$ & $60(30 \%)$ & 0.13 \\
\hline Triptans & $129(26 \%)$ & $25(13 \%)$ & $<0.001$ \\
\hline Ergots & 119 (24\%) & 66 (33\%) & 0.02 \\
\hline Opioids & $5(1 \%)$ & $6(3 \%)$ & 0.31 \\
\hline Poly-overuse & $37(8 \%)$ & $14(7 \%)$ & 0.87 \\
\hline Drug days per month & $22.0(6.4)$ & $22.6(6.6)$ & 0.33 \\
\hline MIDAS & $59.9(51.2)$ & $59.8(58.6)$ & 0.99 \\
\hline HADS depression & $6.5(3.8)$ & $7.1(4.1)$ & 0.06 \\
\hline HADS anxiety & 9.1 (4.8) & $10.0(4.8)$ & 0.03 \\
\hline
\end{tabular}

Data are presented as means with standard deviations or percentages in brackets. $\mathrm{MOH}$ : medication-overuse headache; MIDAS: Migraine Disability Assessment; HADS: Hospital Anxiety and Depression Scale. Each patient could have more than one type of headache, therefore the numbers add up to more than 100\%. Primary headache type was diagnosed according to The International Classification of Headache Disorders, second edition (ICHD-II) (2I) except for chronic migraine, which was diagnosed according to the appendix criteria (19).

The following results are presented for the 492 patients who completed the study.

\section{Headache days and days with intake of analgesics}

In total, headache days were reduced by $58.5 \%$ from 23.6 (6.1) to $9.8 \quad(7.8)$ days/month $(p<0.001)$. Medication intake was reduced by $70.0 \%$ from 22.0 (6.4) to $6.6(5.7)$ days/month $(p<0.001)$. All patients completed the detoxification protocol at month 2, i.e. did no longer overuse medications, while $32(6.5 \%)$ had relapsed to $\mathrm{MOH}$ at six months' follow-up.

\section{Disability}

The average MIDAS score was significantly reduced by $57.1 \%$ from baseline $59.9(51.7)$ to $25.7(40.5)$ at six months after detoxification $(p<0.001)$. The scores for all five questions were each reduced by more than $50 \%$, covering the domains: loss of work or school days, number of days with more than $50 \%$ reduction in productivity, number of days not being able to do household work, number of days with more than $50 \%$ reduction in ability to do household work, and number of days not being able to perform family, social or non-work activities (Figure 2). The degree of disability was dramatically shifted from severe to minimal or mild after detoxification. At baseline, the number of severely disabled patients was 367 (74.6\% of all participants) and after detoxification this number was reduced by $52.6 \%$ to 174 patients $(35.4 \%$ of all participants) and in parallel the number of only minimally disabled patients was increased by $376.9 \%$ from 39 to 186 (Figure 3).

The MIDAS score at baseline was significantly lower in the 315 patients who had more than $50 \%$ reduction in headache days after six months than in the 177 patients who experienced less than $50 \%$ reduction in headache days after six months 55.1 (50.3) vs 69.9 


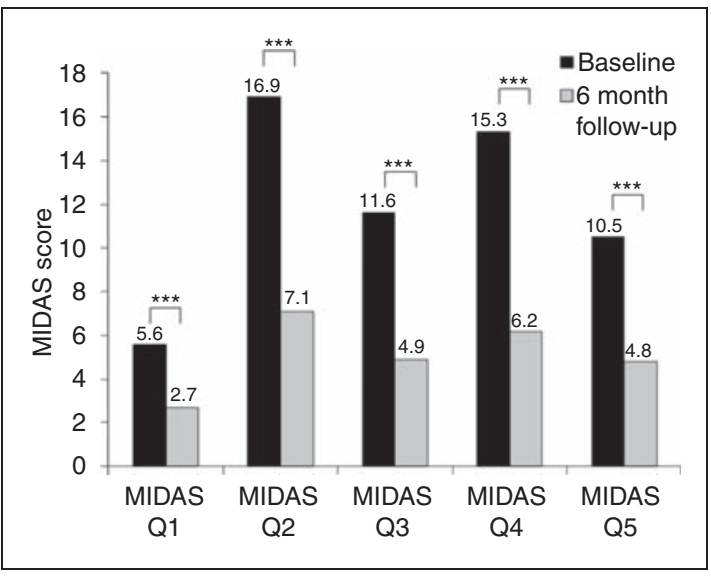

Figure 2. The scores for each of the five questions of the MIDAS questionnaire were significantly reduced from baseline to six months after detoxification $(p<0.00 \mathrm{I})$. Each question represents the number of days with disability caused by headaches within the previous three months. MIDAS QI: Days of work or school missed because of headaches. MIDAS Q2: Days with productivity reduced to less than half at work or school because of headaches. MIDAS Q3: Days with no household work because of headaches. MIDAS Q4: Days with household productivity reduced to less than half because of headaches. MIDAS Q5: Days with family, social or leisure activities missed because of headaches.

$* * * p<0.00$ I. MIDAS Q I-5: Migraine Disability Assessment Questions one through five.

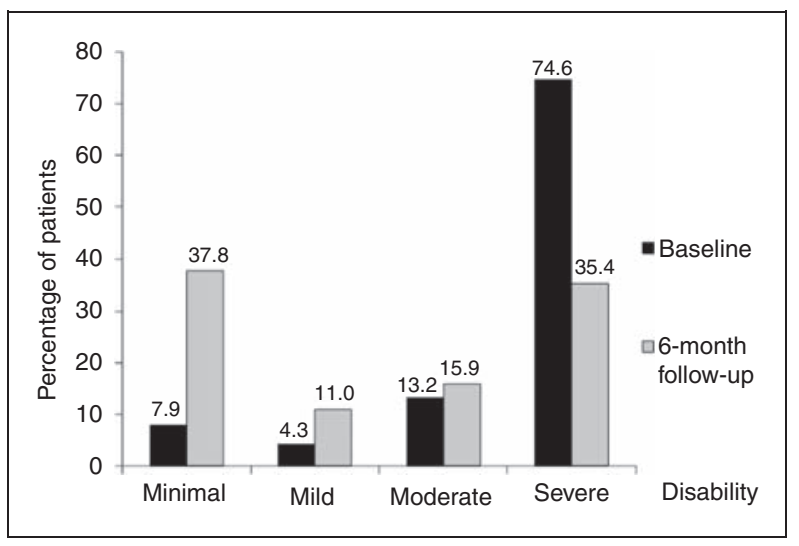

Figure 3. Percentage of patients suffering from minimal, mild, moderate and severe disability, as calculated according to Migraine Disability Assessment (MIDAS) score, before and six months after detoxification and prophylaxis. The number of patients suffering from severe disability was reduced with $52.6 \%$ after detoxification and prophylaxis, while the number of only minimally disabled patients was increased by $376.9 \% . N=492$.

(55.3), $p=0.003$. Patients with baseline MIDAS score below 60 had a $62.3 \%$ reduction of headache days from baseline, while patients with baseline MIDAS score above 60 had a 51.4\% reduction, both reductions

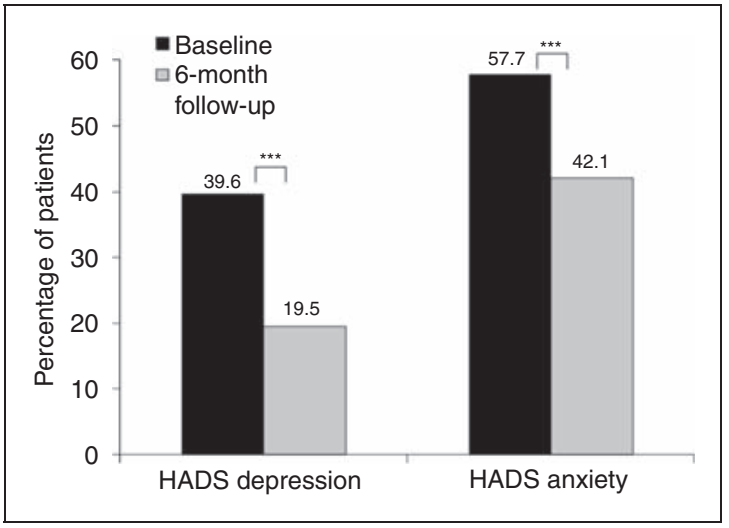

Figure 4. Percentage of patients with depression and anxiety according to the Hospital Anxiety and Depression Scale (HADS) were significantly reduced from baseline to six months after detoxification and prophylaxis.

$N=492$. *** $p<0.001$.

were highly significant $(p<0.001)$, as was the difference between groups $(p<0.001)$. Furthermore, baseline MIDAS score was 58.6 (51.9) in the 460 patients no longer having $\mathrm{MOH}$ after six months and 71.9 (56.5) in those 32 patients still overusing medications at six months $(p=0.08)$.

\section{Depression and anxiety}

According to HADS, a total of $195(40.0 \%)$ patients fulfilled the criteria for depression and $284(57.7 \%)$ for anxiety, i.e. scored more than 8 on the HADS depression or anxiety at baseline. At the end of the study, the number of patients with depression was reduced by $50.8 \%$ from $195(40.0 \%)$ to $96(19.5 \%)(p<0.001)$, while the number of patients with anxiety was reduced with $27.1 \%$ from $284(57.7 \%)$ to $207 \quad(42.1 \%)$ $(p<0.001)$ (Figure 4). HADS depression score was reduced from baseline 6.5 (3.8) to 4.1 (3.7) at six months $(p<0.001)$, while HADS anxiety score was reduced from baseline 9.1 (4.8) to 7.1 (4.4) at six months $(p<0.001)$.

There was no difference in the number of headache days or days with drug intake per month at baseline between subjects who had depression, 23.4 (6.2) headache days and 21.9 (6.6) drug days and those who did not have depression, $23.6(6.0)$ headache days and 22.1 (6.3) drug days $(p=0.70$ and $p=0.68)$. The effect of detoxification was highly significant in both groups. Those without depression at baseline had a slightly better outcome after six months with a $62.3 \%$ reduction in headache days to 8.9 (7.1) headache days/month and $71.9 \%$ reduction in drug days to 6.2 (5.2) drug days/ month compared with those with depression at baseline who after six months had a $52.1 \%$ reduction in headache days to 11.2 (8.4) headache days/month and a 
$66.7 \%$ reduction in drug days to 7.3 (6.4) drug days/ month ( $p=0.002$ and $p=0.13$ ).

At baseline, there was no difference in number of headache days between subjects who had anxiety 23.6 (6.2) days and those who did not have anxiety 23.4 (6.0) days $(p=0.58)$. Patients with anxiety had 22.5 (6.4) drug days at baseline, which was slightly more than 21.3 (6.3) drug days for patients who did not have anxiety $(p=0.046)$. The effect of detoxification was highly significant in both groups. After six months, patients with anxiety at baseline had a $58.5 \%$ reduction in headache days to 9.8 (7.9) headache days/month and a $69.3 \%$ reduction in drug days to 6.9 (6.2) drug days/month, while patients without anxiety had a $58.1 \%$ reduction in headache days to 9.8 (7.6) headache days/month and a $70.4 \%$ reduction in drug days to 6.3 (5.1) drug days/month. There was no difference in reduction of headache days ( $p=0.84)$ or drug days $(p=0.55)$ between the groups.

\section{Discussion}

The major finding in this large multicentre, multinational treatment study was that disability can be considerably reduced by detoxification and prophylactic treatment in patients with $\mathrm{MOH}$. Importantly, evaluation was not performed shortly after treatment but after six months, suggesting that this will be a longterm effect in most patients. Headache days and days with medication intake were considerably reduced in these patients.

It is also very important to note that the baseline MIDAS score was very high in our group of $\mathrm{MOH}$ patients, indicating a high degree of disability. This is in agreement with previous studies (2,7-11). A recent study from a tertiary headache centre in Germany reported that patients with $\mathrm{MOH}$ had considerably higher MIDAS and HADS scores than patients with migraine and tension-type headache (8). In this study, MIDAS score for $69 \mathrm{MOH}$ patients was 70.5 compared with 39.5 for 62 migraine patients (8).

We found impressive improvements in disability after detoxification and prophylaxis with $57.1 \%$ total reduction in MIDAS and more than $50 \%$ reduction in all individual domains, indicating a highly significant improvement in disability and reduction of burden of headache in the individual patient. This is underscored by the fact that the degree of disability was dramatically shifted from severe to minimal or mild after detoxification and prophylaxis with $52.6 \%$ reduction in the number of severely disabled patients. To our knowledge only a few previous studies have investigated long-term effects of detoxification and prophylaxis upon disability in $\mathrm{MOH}$. In the previously mentioned German study (8), MIDAS score was reduced from 53 to 37 after six months of treatment as a total for all groups of patients, but specific figures for $\mathrm{MOH}$ were not provided. Our results are in line with a previous study from Italy, where Andrasik et al. reported considerable reductions in MIDAS following treatment in $84 \mathrm{MOH}$ patients. MIDAS scores were reduced from 70.8 before to 23.3 at six months and 34.1 at 12 months after treatment. After three years and five years, MIDAS scores were still low and at the same level as after six months $(11,21)$. This demonstrates a very positive long-term effect on disability by treatment of $\mathrm{MOH}$ and indicates that disability results after six months can be used as a predictor for long-term outcome.

A large proportion of our patients were defined as being depressed or anxious when using the cut-off score of 8 on the HADS, and our patients had rather high depression and anxiety scores at baseline. The number of depressed patients and the HADS depression score were considerably reduced by detoxification and prophylaxis in the present study, while the effect of our intervention on anxiety was more modest. Wallasch and Kropp found comparable figures of HADS depression scores of 6.4 before treatment but a lower HADS anxiety score of 7.8 in their sample of $69 \mathrm{MOH}$ patients (8). Reductions in these figures after treatment were not mentioned specifically for $\mathrm{MOH}$. Our findings strongly indicate that the depressive symptoms and, to a lesser extent, anxious symptoms in patients with $\mathrm{MOH}$ are a consequence of the disabling headaches and not a personality disorder per se. Conversely, the present findings also stress the importance of a precise history of headache and probably also other pain disorders and intake of pain medications in depressed patients.

Zidverc-Trajkovic et al. previously reported high MIDAS score at baseline as a predictor for poor outcome (10). The 137 patients in their study who had more than $50 \%$ reduction in headache after detoxification and prophylaxis had lower baseline MIDAS scores than the 103 patients who had less than $50 \%$ reduction in headache. With the present data we further confirm the negative predicting value of a high baseline MIDAS score for the outcome of $\mathrm{MOH}$. However, it is noteworthy that even those of our patients with the highest MIDAS scores, arbitrarily set as a MIDAS score above 60 , had a pronounced improvement in headache after detoxification and prophylaxis. Thus, all patients regardless of baseline MIDAS should be offered a detoxification programme.

Hagen et al. reported that a low total HADS score at baseline was associated with a favourable outcome after treatment in $50 \mathrm{MOH}$ patients, while Bøe et al. did not confirm this in a sample of $80 \mathrm{MOH}$ patients $(3,18)$. We found a significantly better outcome 
in terms of headache days after six months in $\mathrm{MOH}$ patients without depression according to HADS, while the presence of anxiety did not predict outcome. However, the absolute differences in outcome between our patients with and without depression were quite small compared with the impressive reductions in headache days found in both groups.

Thus, it can be concluded that treatment of $\mathrm{MOH}$ according to the proposed protocol is highly effective and should be performed in all patients regardless of the degree of disability, depression and anxiety.

\section{Methodological considerations}

A major strength of the study is the large group of patients, the multinational design and the follow up time of six months.

A limitation of the present study is the lack of a control group. However, the patients had had chronic headache for more than five years on average and therefore a dramatic improvement within six months would be unlikely. Inclusion of a control group would be desirable but it would also pose ethical problems to withhold effective treatment from patients who often have already been waiting for quite some time before being admitted to a headache centre.

Diagnosis of $\mathrm{MOH}$ requires that the headache has developed or markedly worsened during medication overuse. Since $\mathrm{MOH}$ patients usually have had chronic headache and $\mathrm{MOH}$ for several years, in this study for four to five years, this criterion as well as estimations of duration of chronic headache and $\mathrm{MOH}$ may be subject to recall bias. However, since it was impossible to collect headache diaries from all patients for several years before inclusion in this study, a thorough history from the patients was the best possible method to obtain these data. Therefore, information about the primary headache type always needs to be considered with caution.

Since patients were managed both by detoxification and preventive medications, this study does not clarify the relative importance for each of these therapies on the positive outcome.

Non-completers had similar MIDAS score as completers at baseline, but they included a lower percentage of triptan overusers, a higher percentage of ergot users and slightly higher HADS scores as compared with completers. This indicates that non-completers could be more difficult to manage than completers. The present results may therefore not be representative for all $\mathrm{MOH}$ patients.

\section{Conclusions}

Disability, depression and anxiety were considerably reduced in patients with $\mathrm{MOH}$ by the proposed treatment protocol. This was true regardless of the degree of disability, depression and anxiety before management. These results emphasise the urgent need for increased awareness about avoiding overuse of headache medications both among the public and professionals in order to prevent medication overuse. Similarly, the dramatic and positive effect on a chronic disabling pain disorder such as $\mathrm{MOH}$ demonstrates the need for structured treatment programmes for $\mathrm{MOH}$ as not only headache days but also disability are remarkably improved by detoxification and prophylactic treatment.

\section{Clinical implications}

- Disability, depression and anxiety can be considerably reduced in patients with medication-overuse headache $(\mathrm{MOH})$ by detoxification and prophylactic treatment.

- There is an urgent need for increased awareness about avoiding overuse of headache medications both among the public and professionals.

- Not only headache days but also disability are remarkably improved by adequate intervention in patients with $\mathrm{MOH}$.

\section{Funding}

This work was supported by the COMOESTAS Project - EC contract number 215366 (COMOESTAS) FP7 - Thematic priority ICT.

\section{Conflict of interest}

None declared.

\section{References}

1. Linde M, Gustavsson A, Stovner LJ, et al. The cost of headache disorders in Europe: The Eurolight project. Eur J Neurol 2012; 19: 703-711.

2. Munksgaard SB, Bendtsen L and Jensen RH. Treatmentresistant medication overuse headache can be cured. Headache 2012; 52: 1120-1129. 
3. Hagen K, Albretsen C, Vilming ST, et al. A 4-year follow-up of patients with medication-overuse headache previously included in a randomized multicentre study. J Headache Pain 2011; 12: 315-322.

4. Grande RB, Aaseth K, Benth JS, et al. Reduction in medication-overuse headache after short information. The Akershus study of chronic headache. Eur J Neurol 2011; 18: 129-137.

5. Rossi P, Di Lorenzo C, Faroni J, et al. Advice alone vs. structured detoxification programmes for medication overuse headache: A prospective, randomized, openlabel trial in transformed migraine patients with low medical needs. Cephalalgia 2006; 26: 1097-1105.

6. Munksgaard SB, Bendtsen L and Jensen RH. Detoxification of medication-overuse headache by a multidisciplinary treatment programme is highly effective: A comparison of two consecutive treatment methods in an open-label design. Cephalalgia 2012; 32: 834-844.

7. Lanteri-Minet M, Duru G, Mudge M, et al. Quality of life impairment, disability and economic burden associated with chronic daily headache, focusing on chronic migraine with or without medication overuse: A systematic review. Cephalalgia 2011; 31: 837-850.

8. Wallasch TM and Kropp P. Multidisciplinary integrated headache care: A prospective 12-month follow-up observational study. J Headache Pain 2012; 13: 521-529.

9. Raggi A, Giovannetti AM, Leonardi M, et al. Disability and mood state in patients with episodic and chronic migraine associated to medication overuse. Neurol Sci 2012; 33(Suppl 1): S169-S171.

10. Zidverc-Trajkovic J, Pekmezovic T, Jovanovic Z, et al. Medication overuse headache: Clinical features predicting treatment outcome at 1-year follow-up. Cephalalgia 2007; 27: 1219-1225.

11. Andrasik F, Grazzi L, Usai S, et al. Disability in chronic migraine with medication overuse: Treatment effects through 5 years. Cephalalgia 2010; 30: 610-614.
12. Stewart WF, Lipton RB, Kolodner K, et al. Reliability of the migraine disability assessment score in a populationbased sample of headache sufferers. Cephalalgia 1999; 19: 107-114.

13. Stewart WF, Lipton RB, Dowson AJ, et al. Development and testing of the Migraine Disability Assessment (MIDAS) Questionnaire to assess headache-related disability. Neurology 2001; 56: S20-S28.

14. Edmeads J, Láinez JM, Brandes JL, et al. Potential of the Migraine Disability Assessment (MIDAS) Questionnaire as a public health initiative and in clinical practice. Neurology 2001; 56: S29-S34.

15. Bjelland I, Dahl AA, Haug TT, et al. The validity of the Hospital Anxiety and Depression Scale. An updated literature review. J Psychosom Res 2002; 52: 69-77.

16. Maizels M, Smitherman TA and Penzien DB. A review of screening tools for psychiatric comorbidity in headache patients. Headache 2006; 46(Suppl 3): S98-S109.

17. Peng KP and Wang SJ. Migraine diagnosis: Screening items, instruments, and scales. Acta Anaesthesiol Taiwan 2012; 50: 69-73.

18. Bøe MG, Salvesen R and Mygland A. Chronic daily headache with medication overuse: Predictors of outcome 1 year after withdrawal therapy. Eur J Neurol 2009; 16 : $705-712$.

19. Olesen J, Bousser MG, Diener HC, et al. New appendix criteria open for a broader concept of chronic migraine. Cephalalgia 2006; 26: 742-746.

20. Headache Classification Subcommittee of the International Headache Society. The International Classification of Headache Disorders: 2nd edition. Cephalalgia 2004; 24(Suppl 1): 1-160.

21. Andrasik F, Grazzi L, Usai S, et al. Disability in chronic migraine with medication overuse: Treatment effects at 3 years. Headache 2007; 47: 1277-1281. 